\title{
Decompressive craniectomy for acute stroke: the good, the bad, and the ugly of it
}

\author{
Dhaval Shukla ${ }^{1}$, Amit Agrawal $^{2}$ \\ ${ }^{1}$ Additional Professor of Neurosurgery, Department of Neurosurgery, National Institute of \\ Mental Health and Neurosciences, Bangalore, Karnataka (India) \\ ${ }^{2}$ Professor of Neurosurgery, Department of Neurosurgery, Narayana Medical College Hospital, \\ Chinthareddypalem, Nellore, Andhra Pradesh (India)
}

\begin{abstract}
Large hemispheric infarctions have malignant course and constitute a major cause of severe morbidity and mortality after stroke. The medical management is usually not effective in these cases. Decompressive craniectomy is a salvage therapy for medically refractory ICP. This paper discusses the merits and demerits of decompressive craniectomy for large hemispheric infarctions. Hemicraniectomy is a life-saving but nonrestorative surgery. Surgery should be done before clinical signs of brain herniation to obtain maximum benefit. The relatives of the patient should be explained clearly about possibility of survival with disability before offering the surgery.
\end{abstract}

Key words: hemispheric infarctions, decompressive craniectomy, hemicraniectomy, brain herniation, stroke.

\section{Introduction}

Large hemispheric infarctions have malignant course and constitute a major cause of severe morbidity and mortality after stroke.
It is usually due to occlusion of distal internal carotid artery (ICA) or proximal middle cerebral artery (MCA) trunk without sufficient collateral flow. Atrial fibrillation and ICA dissection are major causes of these occlusions. [6] The incidence of malignant cerebral infarction is 10 to 20 per 100000 per year. [2] Neurological deterioration due to edema occurs in $10 \%$ of all strokes. Neurological decline occurs in $36 \%$ patients within $24 \mathrm{hrs}$ and in $68 \%$ within $48 \mathrm{hrs}$. [2] The first sign of neurological decline is nausea and vomiting followed by drowsiness, and pupillary asymmetry. If not aggressively treated death occurs within 5 days in 41 to $79 \%$ patients.[8] The clinical predictors of malignant infarction are: younger age, female gender, absence of history of previous stroke, systolic BP $>180 \mathrm{mmHg}$ during first 12 hours, and National Institutes of Health Stroke Scale (NIHSS) score $>20$. The radiological predictors are: hypodensity in MCA territory CT $>50 \%$, ICA bifurcation occlusion, involvement of additional vascular territories, hyperdense MCA sign, perfusion deficit of 
more than $66 \%$ of MCA territory on CT scan performed within 6 hours of stroke, and diffusion weighted imaging (DWI) volume on MRI more than $145 \mathrm{ml}$ within $14 \mathrm{~h}$ of stroke onset. [2] The medical management is usually not effective in these cases. Intravenous thrombolysis is less likely to reperfuse large cerebral artery occlusions. The patients require management of raised intracranial pressure (ICP), which include: endotracheal intubation, mechanical ventilation, sedation, and medical management of cerebral edema. The addition of hypothermia is also not much beneficial, and mortality is still high at $43-47 \%$. [8]

\section{Decompressive Craniectomy}

Decompressive craniectomy (DC) is a salvage therapy for medically refractory ICP. It consists of removal of large bone flap over frontal, temporal, and parietal lobe at infarct site. As DC for infarct is usually unilateral i.e. on the side of infarct, it is called as hemicraniectomy. The basic steps of hemicraniectomy are following: [7]

Skin incision: A large reverse question mark skin incision is made. The incision begins 2 to $3 \mathrm{~cm}$ lateral to midline behind the hairline, extends at least $15 \mathrm{~cm}$ posteriorly, and then curves around and down to the posterior root of zygoma. The skin and temporalis muscle are reflected anteriorly as a myocutaneous flap.

Bone removal: The limits of bone removal are $2-3 \mathrm{~cm}$ from midline, avoiding frontal sinus and superior sagittal sinus, till middle cranial fossa base. The anteroposterior extent is atleast $12 \mathrm{cms}$.

Dural opening:The dura is opened in a stellate fashion to maximize cerebral decompression. The bulging brain can be covered with pericranium or dural substitute for augmented duraplasty. DC helps by reversing brain shifts, and relieving ventricular and vascular compression. This allows edematous brain to expand extracranially instead of compressing the normal opposite cerebral hemisphere and brain stem. Hemicraniectomy is also believed to improve cerebral perfusion pressure (CPP), increase retrograde flow in MCA, preserve cerebral blood flow (CBF), andprevent further ongoing ischemia.

\section{Good about DC}

The outcome of DC is quoted from French DECIMAL (DEcompressiveCraniectomy In MALignant middle cerebral-artery infarcts), German DESTINY (DEcompressive Surgery for the Treatment of malignant INfarction of the middle cerebral arterY), and Dutch HAMLET (Hemicraniectomy After Middle cerebral artery infarction with Lifethreatening Edema Trial) trials. [4] The survival is improved from $29 \%$ with medical treatment to $78 \%$ resulting in absolute risk reduction ARR of $50 \%$. Among survivors the good functional outcome (modified Rankin Scale (mRS) score $\leq 4$ ) is increased from $24 \%$ to $75 \%$ with ARR of $51 \%$. The number needed to treat (NNT) is 2 for survival. [4] Hemicraniectomy is lifesaving surgery in patients with malignant hemispheric infarction.

\section{Bad about DC}

The outcome discussions are often dichotomized into simply survival or death. The above mentioned trial included a mRS 
DOI: 10.2478/romneu-2014-0048

score of 4 (moderately severe disability; unable to walk without assistance and unable to attend to own bodily needs without assistance) as a favourable outcome, hence apparent result of these trials showed good number of patients with favorable outcome. Such a class of mRS should not be clubbed with patients with good outcome. The NNT for survival with better function defined as $\mathrm{mRS} \leq 3$ (moderate disability; requiring some help, but able to walk without assistance) is 4 , i.e. out of 4 patients who survive after surgery only 1 will have good functional outcome. [5] Including mRS 4 about $40 \%$ of survivors have severe disability. Very little attention is paid about disability after surgery. There is very limited time prior to surgery for patients to understand the benefits and outcome of DC. The decision to operate is not always made in the light of the real probabilities of surviving with severe disability. [5] The patients are not prepared to accept the risk of unacceptable badness after surgery. The operating neurosurgeon and referring neurologist, before hey consign a patient to survival with a severe disability, have an obligation to establish that this would have been acceptable to them. [5] The American HeADDFIRST study (Hemicraniectomy and Durotomy Upon Deterioration from Infarction Related Swelling Trial) and Philippine HeMMI trial (Hemicraniectomy For Malignant Middle Cerebral Artery Infarcts) have included quality of life as a outcome measure and will give more answers to this problem of disabled survivors. [8]

Another problem with DC is delayed mortality. Most of the patients who are manged medically die during initial few days.
The preliminary results of HeADDFIRST study showed that mortality after DC approximated to that of medical treatment after 6 months. This delayed mortality may be due to complications associated with DC. [8]

The timing of surgery has mixed results, with some studies showing that early surgery decreases mortality, others reporting no difference or worse outcomes. The functional outcome is uncertain in the studies dealing with timing of surgery. The results of HAMLET show that surgical decompression within 4 days of symptom onset does not reduce poor outcome in patients. As many of our patients are referred late after neurological deterioration and development of clinical signs of brain herniation, we can offer a little by operating upon these patients. [4]

The benefit of surgery in older individuals is also not clear. In an earlier study, though the mortality was reduced, none of survivors have a Barthel Index (BI) score $>60$ or a mRS score $<$ 4. A recent study showed no significant difference between mortality. The NIHSS score before surgery contributed to the sixmonth mortality. Hence when surgery is offered to elderly patients factors other than age should be considered and the treatment should be individualized in elderly patients. An upcoming DESTINY-II trial will study patients older than 60 years and will clarify the issues of surgery for elderly population. [8]

\section{Uglyabout DC}

Hemicraniectomy sounds a very simple surgical procedure, but is not without complications. None of the above mentioned studies discussed about complications of DC in detail. The incidence of any complication after DC is $50 \%$ to $55 \%$. [3] The complications 
include hemorrhagic infarction, necrosis, hematomas and edema at the site of the craniectomy in about 25\% cases. [3] Paradoxical cerebral oedema after DC is attributed to possible reduction in the interstitial pressure within the brain after decompression, resulting in a greater hydrostatic pressure gradient between the intravascular and interstitial spaces. Other complications are infection and seizures in 7$20 \%$ cases. [3] The delayed complications are cerebrospinal fluid absorption disorders including subdural hygroma in $6-21 \%$ and hydrocephalus in $10-40 \%$ cases. [3] A complciation specific to DC is syndrome of the trephined (also called as sunken flap syndrome) which is due to subatmospheric pressure, irritation of neural parenchyma at bone edges, and adhesion of brain to subcutaneous tissue. The clinical manifestation of this syndrome is headache, seizures, mood swings, and behavioral disturbances. This syndrome can be prevented by early bone flap replacement (cranioplasty). [3]

Cranioplasty is filling of skull defect. This is usually performed after 6 weeks to 6 months of surgery. This procedure is also not without complications, which are reported in about $34 \%$ cases. [1] The common complications after cranioplasty are infection, wound breakdown, intracranial hemorrhage, bone resorption, and sunken cranioplasty. There is no difference in infection rate irrespective of autologous/ allogenic (bone substitutes, titanium) material used, method of bone flap preservation, and timing of replacement of bone flap. [1]

\section{Conclusion}

Hemicraniectomy is a life-saving but nonrestorative surgery. Surgery should be done before clinical signs of brain herniation to obtain maximum benefit. The relatives of the patient should be explained clearly about possibility of survival with disability before offering the surgery.

\section{Correspondence}

Dr. Dhaval Shukla (MCh)

Additional Professor of Neurosurgery

Department of Neurosurgery

National Institute of Mental Health and

Neurosciences

Bangalore

Karnataka (India)

Email-neurodhaval@rediffmail.com

Phone- +91-8026995411

\section{References}

1.Gooch MR, Gin GE, Kenning TJ, German JW. Complications of cranioplasty following decompressivecraniectomy: analysis of 62 cases. Neurosurg Focus. 2009;26:E9.

2.Hofmeijer J, Algra A, Kappelle LJ, van der Worp HB. Predictors of life-threatening brain edema in middle cerebral artery infarction. Cerebrovasc Dis. 2008;25:176184.

3.Honeybul S, Ho KM. Long-term complications of decompressive craniectomy for head injury. J Neurotrauma. 2011;28:929-935.

4.Huttner HB, Schwab S. Malignant middle cerebral artery infarction: clinical characteristics, treatment strategies, and future perspectives. Lancet Neurol. 2009;8:949-958.

5.Kelly AG, Holloway RG. Health state preferences and decision-making after malignant middle cerebral artery infarctions. Neurology. 2010;75:682-7.

6.Kimberly WT, Sheth KN. Approach to severe hemispheric stroke. Neurology. 2011;76(Suppl 2):S50-6. 7.Staykov D, Gupta R. Hemicraniectomy in malignant middle cerebral artery infarction. Stroke. 2011;42:513-6. 8.Wartenberg KE. Malignant middle cerebral artery infarction. Curr Opin Crit Care. 2012;18:152-63. 